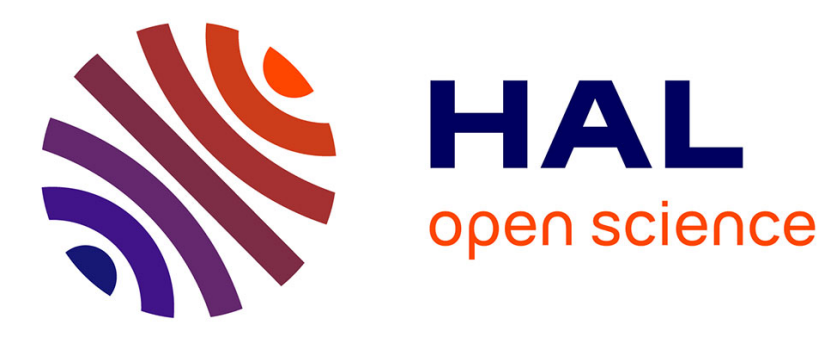

\title{
First-principles assessment of potential ultrafast laser-induced structural transition in $\mathrm{Ni}$
}

Emile Bévillon, Jean-Philippe Colombier, Razvan Stoian

\section{To cite this version:}

Emile Bévillon, Jean-Philippe Colombier, Razvan Stoian. First-principles assessment of potential ultrafast laser-induced structural transition in Ni. Applied Surface Science, 2016, E-MRS 2015 Spring Meeting Symposium CC: "Laser and plasma processing for advanced applications in material science", 11-15 May 2015, Lille (France), 374, pp.365-369. 10.1016/j.apsusc.2015.12.139 • ujm-01340987

\section{HAL Id: ujm-01340987 \\ https://hal-ujm.archives-ouvertes.fr/ujm-01340987}

Submitted on 8 Jul 2016

HAL is a multi-disciplinary open access archive for the deposit and dissemination of scientific research documents, whether they are published or not. The documents may come from teaching and research institutions in France or abroad, or from public or private research centers.
L'archive ouverte pluridisciplinaire HAL, est destinée au dépôt et à la diffusion de documents scientifiques de niveau recherche, publiés ou non, émanant des établissements d'enseignement et de recherche français ou étrangers, des laboratoires publics ou privés. 


\title{
Applied Surface Science
}

Volume 374, 30 June 2016, Pages 365-369

E-MRS 2015 Spring Meeting Symposium CC: "Laser and plasma processing for advanced applications in material science", 11-15 May 2015, Lille (France)

\section{First-principles assessment of potential ultrafast laser-induced structural transition in $\mathrm{Ni}$}

\author{
E. Bévillon, ${ }^{1}$ J.P. Colombier, ${ }^{1, *}$ and R. Stoian $^{1}$ \\ ${ }^{1}$ Laboratoire Hubert Curien, UMR CNRS 5516, Université de Lyon, \\ Université Jean-Monnet 42000, Saint-Etienne, France
}

\begin{abstract}
The possibility to trigger ultrafast solid-to-solid transitions on transition metals under femtosecond laser irradiation is investigated by means of first-principles calculations. Electronic heating can drastically modify screening, charge distribution and atomic binding features, potentially determining new structural relaxation paths. Consequently, we evaluate here the effect of electronic excitation on structural stability and conditions for structural transitions. $\mathrm{Ni}$ is chosen as a case study, and the stability of its FCC phase is compared to the non-standard HCP structure while accounting for the heating of the electronic subsystem. From a phonon spectra analysis, we show that the thermodynamic stability order reverse at an electronic temperature of around $10^{4} \mathrm{~K}$. Both structures exhibit a dynamic stability, indicating they present a reversible stability or a metastability depending on the heating. However, the general hardening of phonon modes with the increase of the electronic temperature points out that no transformation will occur, as confirmed by the study of a typical FCC to HCP diffusionless transformation path, showing an increasing energy barrier. Finally, based on electronic density of states interpretation, the tendency of different metal categories to undergo or not an ultrafast laser-induced structural transition is discussed.
\end{abstract}

\section{INTRODUCTION}

The dynamic response of materials subject to femtosecond laser irradiations is an area of intense activities ${ }^{1-3}$. Particularly the understanding of the primary excitation events and energy transport is crucial as they condition the energy absorption, storage and release, and thus subsequent phenomena as ablation and surface nanostructuring. In this context, the knowledge of nonthermally driven phase transitions is crucial as it may rapidly modify thermomechanical, optical or magnetic properties. For instance, in semiconductors, the electronic excitation rapidly weaken the covalent bonding leading to global lattice instability and ultrafast melting ${ }^{4}$. Recently, a related phenomena was shown on half-filled transition metals, the photoexcitation inducing local lattice softenings with potential consequences on ultrafast solid-to-solid transition ${ }^{5}$. Beside this, an accurate description is necessary to correctly describe ultrafast structural dynamics ${ }^{2,6}$, phase transitions ${ }^{7}$, nanostruture formation $^{8}$, ablation dynamics ${ }^{9,10}$, or strong shock propagation $^{11}$. The interplay between ultrafast excitation and resulting excited material response still requires a comprehensive theoretical description for laser-heated materials in solid state.

Prior any subsequent energy dissipations, femtosecond laser irradiations rapidly heat the electronic subsystem of

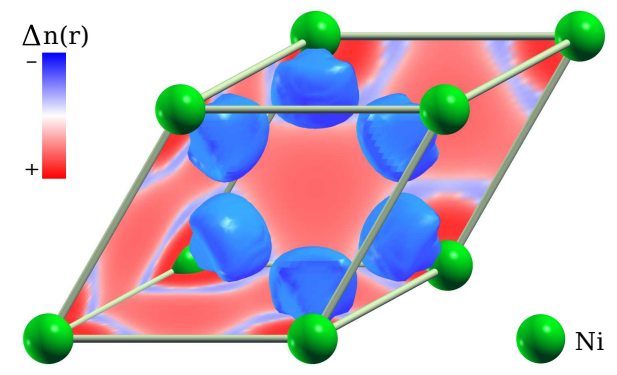

FIG. 1. (Color online) Representation of the electronic density changes from $T_{e}=0 \mathrm{~K}$ to $T_{e}=2.5 \times 10^{4} \mathrm{~K}$, for a primitive cell of FCC Ni. In blue, isosurface of electronic density loss. In red, mapping along basal planes of the electronic density gain.

a metal, inducing significant redistributions of the electronic density. This is illustrated in the case of $\mathrm{Ni}$ in Fig 1, assuming a thermalized state of the electrons ${ }^{12-14}$. The difference of electronic density determined at two different temperatures on $\mathrm{Ni}$ metal shows that a spatially localized volume is impacted by electronic density loss while the rest of the system gains electronic density. This observation illustrates concepts involving the change of the localization degree of the electronic density upon laser heating of the electronic subsystem ${ }^{15}$. In these conditions, the material undergoes an electron-phonon 
nonequilibrium having consequences on thermodynamic and optical properties ${ }^{15-17}$, that modify in turn the energy deposition, storage and its further release. It also induces strong electronic pressures as well as nonthermal forces at very short time scales, having the potentiality to induce ultrafast phase transitions ${ }^{4}$ or solid-to-solid transformations ${ }^{5}$.

The potentiality of an ultrafast generation of dynamic instabilities leading to solid-to-solid diffusionless transformations have already been investigated by Giret et $a l^{5}$ on transition metals. The authors demonstrated the local softening of $\mathrm{W}$ phonon modes upon electronic excitation, corresponding to specific lattice instabilities that affect the potential energy surface. Accordingly, the energy barriers separating the BCC and FCC structures from one side, and the BCC and HCP structures on the other side, are found to soften, potentially leading to ultrafast phase transitions. This phenomenon is related to the formation of nonthermal forces breaking the equilibrium insured by the bonding directionality of half-filled $d$-band metals at standard conditions. In this frame, the noble metals having a filled $d$-band structure, involving a weak directionality of the bonding, are excluded from ultrarapid structural transitions as pointed out by the phonon modes hardening of gold ${ }^{4}$. However, there is still no investigations on almost-filled $d$-band metals, Ni being a typical example of such materials.

Nickel is known to adopt under circumstances an HCP structure, especially in thin films ${ }^{18}$ or during nanoparticles formation ${ }^{19,20}$, showing a propensity to structural changes that might be activated during a laser excitation process. Though the solid-to-solid transformation origin is not involved, we also note the theoretical work of $\mathrm{Wu}$ et $a l^{21}$ pointing out the formation of significant $\mathrm{HCP}$ domains in a normally FCC structure in Ag after a femtosecond laser irradiation, indicating noble metals can also remains in metastable thermodynamic states after laser exposure. The question of potential ultrafast electronic and structural transformations modifying optical, thermal and mechanical properties is a key issue in a range of observations concerning optical coupling. One example concerns the formation of laser-induced periodic structures where the structures periodicities do not match cold material optical indices ${ }^{22,23}$. To elucidate the origin of this disagreement, an ultrafast solidto-solid transition, possibly modifying crystal structures, electronic structures and thus electronic transitions responsible for optical properties, is still an open question.

In this work, to address the conditions of structural changes upon ultrafast excitation, we first evaluate the relative thermodynamic stability of FCC and HCP phases with the electronic temperature. Then, the dynamic stability of both phases is estimated through the analysis of phonon spectra performed along with the excitation process. The energy barrier corresponding to a transformation path connecting both phases is later evaluated. Finally, we discuss the possibility to trigger ultrafast solid-to-solid transition in metals based on the degree of $d$ band filling, and in particular, on changes of the localization of the charge density induced by the rise of the electronic temperature.

\section{CALCULATION DETAILS}

The calculations are carried out with the plane-wave code Abinit ${ }^{24}$, in the frame of the finite temperature density functional theory ${ }^{25-27}$. The exchange and correlation part is modelled within the generalized gradient approximation in the form parameterized by Perdew, Burke and Ernzerhof ${ }^{28}$. The valence electronic configuration of $\mathrm{Ni}$ is $3 d^{8} 4 s^{2}$, nuclei and core electrons are taken into account by Troullier-Martins pseudopotentials ${ }^{29}$. The Monkhorst-Pack method ${ }^{30}$ is employed to mesh the reciprocal space in combination of an energy cutoff for the plane-wave extension of $60 \mathrm{Ha}$. We first performed cell optimization in FCC and HCP structures, providing the respective cell parameters $a_{F C C}=3.55 \AA$ and $a_{H C P}=2.50, c_{H C P}=4.17 \AA$. The experimental value for FCC Ni is $a_{E x p}=3.52 \stackrel{\AA}{A}$, in good agreement with our calculated value.

The effect of intense ultrashort laser irradiation on the electronic subsystem is taken into account by considering a cold lattice and a hot electronic subsystem. This involves that we focus on the first moments of the irradiation when the energy transfer between electrons and lattice is still negligible. The heated electronic subsystem is characterized by an electronic temperature applied through the Fermi-Dirac distribution of the electrons, assuming a fast thermalization of the electrons due to electron-electron collisions, which in metals, is expected to arise around a characteristic time $\tau=10 \mathrm{fs}$ for $T_{e}$ larger than $10^{4} \mathrm{~K}^{12}$. Thus, within the density functional theory, the electronic temperature is taken into account through this electronic distribution, and through an electronic entropy term by minimizing the free energy. This induces a redistribution of the charge density, leading to additional Coulombian forces acting on atoms and modifying material stabilities and dynamics.

\section{RELATIVE THERMODYNAMIC STABILITIES}

The relative stability of FCC and HCP phases with the increase of the electronic temperature is assessed by comparing their free energies. To avoid spurious effects related to non-fully relaxed cell parameters, rigorous cell optimizations were performed for an electronic temperature of $10^{-2} \mathrm{~K}$, leading to residual pressures less than $1 \times 10^{-8} \mathrm{GPa}$ for both phases. It is found that the ionic density is lower for the HCP structure than for the FCC, with volume per atom of 75.62 and 75.36 Bohr $^{3}$ respectively. A focus on the $d$ block electronic structure of both phases is provided in the inset of Fig. 2. The two electronic structures exhibit strong similarities, with however 
a more discontinuous $d$ block for the HCP structure leading to a pseudo band gap at $6.5 \mathrm{eV}$ and a peak of high density on the top of the $d$ block. Although the shape of these $d$ block are globally preserved, the increase of the electronic temperature tends to increase the density of states, to shrink and to shift toward lower energies these $d$ block, independently of the phase, as already observed for noble metals ${ }^{4,15}$.

This impacts the evolution of the free energy with the electronic temperature, and Fig. 2 provides the relative stability of both structures, with the FCC phase considered as a reference and set to $0 \mathrm{eV}$. At low temperature the FCC structure is, as expected, the most stable, but at an electronic temperature of around $10^{4} \mathrm{~K}$ the energy difference vanishes and at higher temperatures the HCP structure becomes more stable. The electronic pressure increases significantly with $T_{e}{ }^{15}$, reaching here around 60 $\mathrm{GPa}$ at $2.5 \times 10^{4} \mathrm{~K}$. This pressure is slightly lowered for the HCP structure, a consequence of the lower ionic density allowing a stronger accommodation of the electronic pressure, providing an additional relaxation mechanism and leading to a more stable phase at high temperature. The stabilization of the HCP phase with the increase of the electronic temperature validates the possibility of a phase transition in nonequilibrium conditions, if confirmed by the material dynamics.

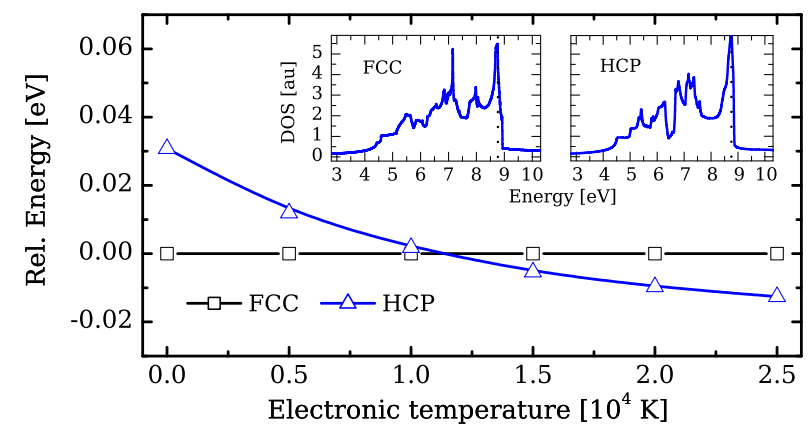

FIG. 2. (Color online) Relative energies of FCC and HCP phases as a function of the electronic temperature. Inset: focus on the $d$ block electronic structure of both phases, computed at $T_{e}=0.01 \mathrm{~K}$.

\section{DYNAMIC STABILITIES}

The dynamic stability of the FCC and HCP phases are investigated within the density functional perturbation approach. For FCC Ni, a $16 \times 16 \times 16 k$-point mesh of the reciprocal space is considered in combination of a $4 \times 4 \times 4 q$-points grid for the calculation of the dynamical matrices used to generate the phonon dispersion curves. For $\mathrm{HCP} \mathrm{Ni}$, the reciprocal and phonon wave vector meshes of $18 \times 18 \times 12$ and $3 \times 3 \times 2$ were considered. The convergence of the results is ensured by tests on the cutoff energy of plane waves, $k$-point and $q$-point grids. The corresponding phonon spectra are provided in Fig. 3 at two different electronic temperatures, 300 and $2.5 \times 10^{4} \mathrm{~K}$. A particularly good agreement is found between theoretical and experimental data of FCC Ni at $300 \mathrm{~K}^{31}$, ensuring the validity of the method. At low temperature, the phonon spectra of both the FCC and HCP phases do not exhibit negative imaginary phonons, indicating they both have a dynamic stability, i.e. a persistence in time. However, considering the relative energies of these phases discussed above, the FCC phase is the most stable, which implies that the HCP phase is metastable.

With the increase of the electronic temperature, a hardening of the phonon modes occurs, pointing out a global hardening of the bondings. This trend was already observed for $\mathrm{Au}^{4}$ and is likely shared with transition metals having filled or almost filled $d$ bands. This phenomena happens for both the FCC and HCP phases, signalling their dynamic stability even at high $T_{e}$. Since the HCP structure becomes more stable at $T_{e}>10^{4} \mathrm{~K}$ (Fig. 2), the stable phase at high temperature is the HCP one whereas the FCC is now metastable, a reversed situation compared to the low temperature case. These results are of interest since they indicate that both phases are thermodynamically stable or metastable and both exhibit dynamic stability, suggesting they could easily coexist within a given time scale. However, the dynamic stability and the hardening of phonon modes implicitly involves the presence of an energy barrier between both phases, which will act as a detriment of the formation of HCP Ni.
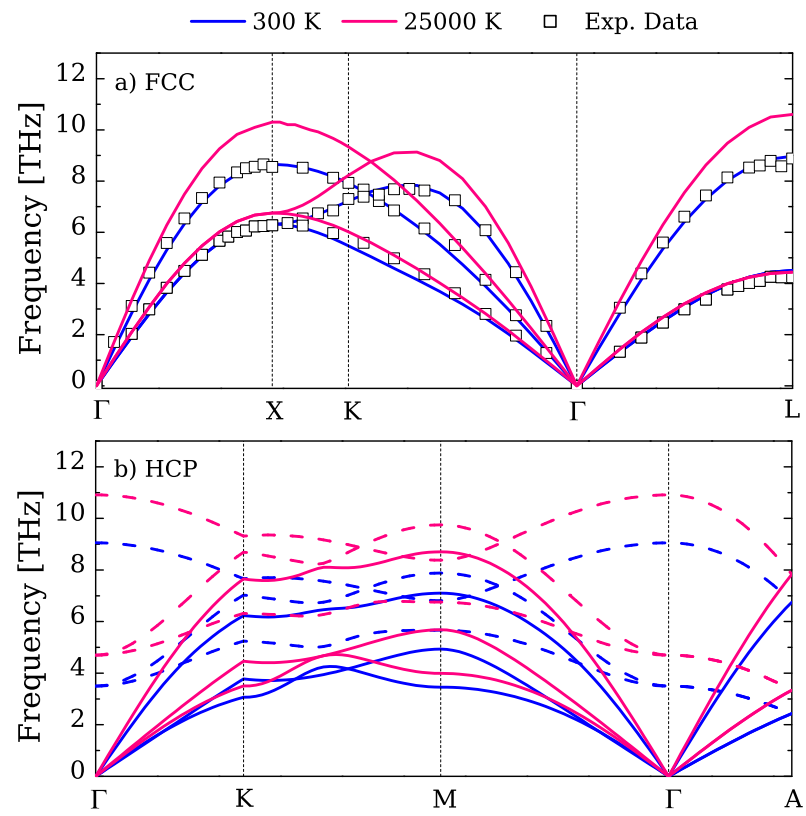

FIG. 3. (Color online) Dispersion curves of phonon as a function of the electronic temperature. a) the FCC phase compared to experimental data and b) the HCP structure. 


\section{STACKING DISORDER GROWTH TRANSFORMATION PATH}

Solid-to-solid transformations belonging to the socalled diffusionless transformations are collective movement of atoms, also called military transformation to highlight the concerted displacement of atoms ${ }^{32}$. These displacements include atomic plan shuffling or latticedistortive displacements, as dilations or shears. They are forbidden in case of an energy barrier separating the initial and final phases, but can be activated in particular conditions of pressure and temperature once the energy barrier soften. A given transformation potentially involves an infinite number of paths leading to the crystal modification. For FCC to HCP transformations, several paths have already been designed ${ }^{33,34}$. Here, we focus on the stacking disorder growth transformation path (SDGTP) that connects a series of ABCABC plans characterizing the FCC structure to the $\mathrm{ABABAB}$ sequence specific of the HCP cell. This occurs through plan shuffling, temporarily vanishing the ordering of stacks responsible of their respective crystal structures. A slight lattice dilation $(+0.4 \%)$ is also applied in order to connect the optimized FCC cell parameters to the HCP ones.

The displacement of atomic plans corresponding to the SDGTP is meshed into a path coordinate, the 0.0 coordinate corresponding to the FCC initial state while the 1.0 coordinate correspond to the HCP final state. The relative free energies corresponding to this path are provided in Fig. 4 at various temperatures. The relative energy differences observed in Fig. 2 are also visible here with a lowering of the HCP energy as $T_{e}$ increase. As suggested by phonon dispersive curves, an energy barrier is observable along with the transformation path. The activation energy, corresponding to the maximum energy needed to activate the displacement is increasing with the rise of the electronic temperature, in agreement with the hardening of phonon modes discussed in previous section. The presence of an energy barrier invalidates the possibility of a collective displacement to occur, and the effect of the electronic temperature does not modify this fact for Ni. Generally speaking, the absence of imaginary mode in the phonon dispersion curves points out that there always be an energy barrier, invalidating potential solid-to-solid transformations from FCC to HCP for Ni.

\section{DISCUSSION}

The energy path connecting both structures can also be discussed in terms of the enthalpy $H=F+P V$, with $F$ the free energy of the system, $V$ the volume of the cell, and $P$ the pressure induced by the rise of the electronic temperature and the cell transformation. At low electronic temperature, and due to the steric hindrance, the hydrostatic pressure increases along the transformation path, reaching its maximum at the saddle position. The

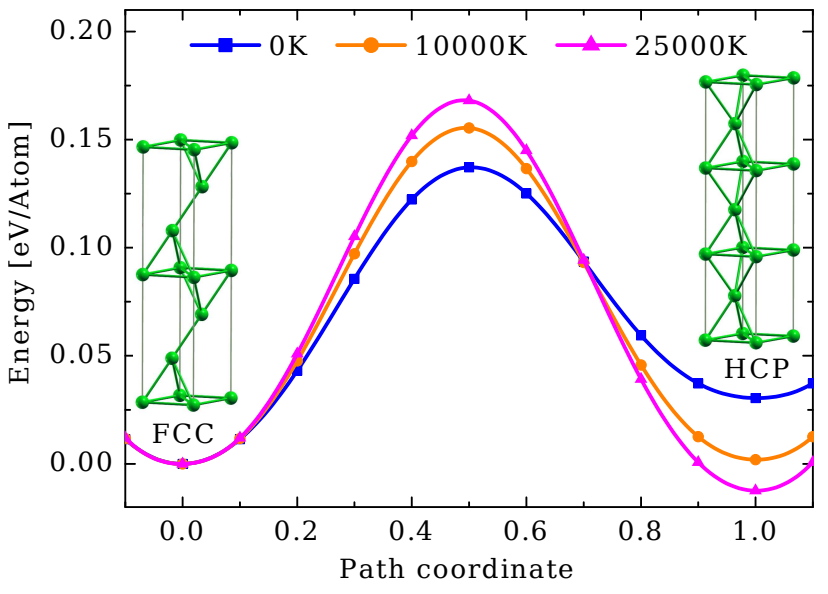

FIG. 4. (Color online) Free energy evolution along the path coordinate connecting the FCC to the HCP structure, at increasing electronic temperatures.

energy contribution of the pressure to the enthalpy quantity produces an enthalpy barrier of $0.46 \mathrm{eV}$ around three times the free energy barrier. As for the free energy barrier, the rise of the electronic temperature induces a slight increase of the enthalpy barrier. Accordingly, considering the enthalpy quantity does not modify the conclusion determined from the analysis of the free energy evolutions. However, it is worth to note that the steric hindering is path dependent, and it is likely that other paths may significantly lower the pressure and thus the computed enthalpy energies. Since the pressure can rapidly reaches tens of $\mathrm{GPa}^{15}$, the corresponding forces rapidly and strongly apply to produce a dilation of the material, having consequences on the cohesion energy or on steric hindrance and possibly modifying the energy and enthalpy barriers. To investigate volume dilation effect, we performed at high temperature $\left(T e \approx 2.5 \times 10^{4} \mathrm{~K}\right)$ the calculation of the transformation path with a $14 \%$ dilated volume corresponding to the liquid density for Ni. This significantly alters the free energy barrier which is reduced to $0.03 \mathrm{eV}$. The enthalpy barrier is lowered by $0.10 \mathrm{eV}$, decreasing from 0.53 to $0.43 \mathrm{eV}$. As a conclusion, the energy and enthalpy barriers remain, confirming the stability of the FCC phase regardless of the electronic heating and related volume dilation.

The Fig. 1 representing electronic density differences between a hot and a cold system, illustrates that important changes of the electronic density localization occur along with the excitation process. From 0.01 to $2.5 \times 10^{4} \mathrm{~K}$ a spatially located area (blue isosurface) is affected by a loss of electronic density. It is located in the vicinity of atoms and oriented toward non-atomic direction, and accordingly, this effect does not affect the bonding. It corresponds to the $T_{e}$ induced depopulation of the top of the $d$ block. A gain of electronic density is observable in the red gradient color of the basal planes. In these mappings, it is possible to distinguish two different red domains, first a weakly red colored area cor- 
responds to the electronic population of the interatomic space, enforcing the metallic bonding of Ni. Secondly, in the vicinity of atoms, a spatially localized area exhibits a significant increase of the electronic density, that is attributed to the bottom part of the $d$ block. This part remains mostly unaffected by electronic depopulation, but the whole $d$ block undergoes a shrinking and shift toward lower energies induced by the strengthening of the effective electron-ion potential ${ }^{15}$, consequence of the decrease of the electronic screening from global $d$ band depopulation. The increase of the electron-ion potential also translates into a shrink of $d$ orbitals, increasing indirectly the electron density in the inner volume initially occupied by the $d$ orbitals, in the vicinity of nuclei. Accordingly, the outer volume of the initial $d$ orbitals are affected by the shrink and exhibits localized loss of electronic density explaining the blue area within the basal planes.

The analysis of the electronic density changes and electronic structure evolution with the electronic temperature is of high interest as it informs about the additional electrostatic forces created during the electronic subsystem heating process. For transition metals having a fully or quasi-fully occupied $d$ block, as Ni or noble metals, the metallic bonding is dominant and the $d$ block is weakly interacting. The electronic heating depopulates the top of the $d$ block, i.e. the less interacting part, depopulating spatially-localized non-bonding $d$ electronic states and populating high-energy spatially-delocalized $s p$ electronic states, enforcing the metallic bonding. The depopulation of the $d$ block leads to an increase of the free electron number but also to a decrease of the electronic screening making the effective electron-ion potential more attractive ${ }^{4,15}$. These phenomena contribute to a hardening of the metallic bonding, as signalled by the hardening of phonon spectra, ensuring the stability of the FCC phase. This explains the hardening of phonon modes as observed on $\mathrm{Au}$ as well ${ }^{4}$. For these metals, the electronic filling of the $d$ block and its non-bonding nature is not dependent on the crystal structure, which explains why the FCC and $\mathrm{HCP}$ phases of Ni reacts similarly to the increase of $T_{e}$.

The case of other metal categories can also be discussed on the lightening of the previous considerations. Simple metals, as Al, do not have a $d$ block, spelectronic states are spatially delocalized and the bonding is metallic. The effect of the electronic heating is to depopulate delocalized electronic states to populate delocalized electronic states. In other words, weak changes are expected either on the metallic nature of such metals and on the electron-ion potential, that should keep thermodynamic and dynamic stabilities. As for Ni or noble metals, solidto-solid transformation are not favourable in such conditions. The category of transition metals having partially filled $d$ block is the most interesting with regard to the possibility to activate solid-to-solid transformations. Despite their metallic nature, they exhibit a directionality in the bonding which is rapidly affected upon electronic excitation, weakening the bonding and generating lattice instabilities, as observed and discussed on $\mathrm{W}$ metal in Ref. ${ }^{5}$. Accordingly, these materials have a propensity to potentially lead to ultrafast solid-to-solid transformation.

\section{CONCLUSION}

In the present work, the potential fcc-to-hcp transition induced by ultrafast laser irradiation is investigated on $\mathrm{Ni}$ metal. The relative energies of both structures as a function of the electronic heating indicate that the thermodynamic stability of phases reverses at $T_{e} \approx 10^{4} \mathrm{~K}$. The phonon modes of both structures do not exhibit a soft mode, indicating that they are dynamically stables, which implies the thermodynamic stability of one and the metastability of the other phase, with a dependency on the electronic heating. The hardening of phonon modes with the electronic temperature points out that an energy barrier separates both structures, invalidating the possibility of a collective displacement of atoms that characterizes the solid-to-solid transformation. This is confirmed by the evolution of the energy variation along a transformation path, showing an increasing energy barrier with $T_{e}$.

These results, in close relation to noble metal observations, point out significant differences with half-filled $d$ band metals as $\mathrm{W}$, with the hardening of phonon modes and the persistence of an energy barrier. From these considerations, and based on an interpretation of metal density of states, the possibility to induce or not a solid transition is discussed. Whereas simple $s p$ metals are not expected to undergo ultrafast transitions, the degree of electronic filling of the $d$ block is considered as being determinant for transition metals. Transition metals having a filled or almost filled $d$ block as $\mathrm{Ni}$ or $\mathrm{Au}$, will not undergo a structural transitions upon ultrafast excitation, while for weakly or partially filled $d$ block ultrafast solidto-solid transitions are likely to occur. This is mainly due to the weak directionality of the $d$ bonds in these metals, that are rapidly affected and weakened by electronic depopulation, possibly leading to lattice instabilities and transitions toward more stable structures.

\section{ACKNOWLEDGMENTS}

This work was supported by the ANR project DYLIPSS (ANR-12-IS04-0002-01) and by the LABEX MANUTECH-SISE (ANR-10-LABX-0075) of the Université de Lyon, within the program "Investissements d'Avenir" (ANR-11-IDEX-0007) operated by the French National Research Agency (ANR). Numerical calculations have been performed using resources from GENCI, project gen 7041 . 
* jean.philippe.colombier@univ-st-etienne.fr

1 K. Sokolowski-Tinten, J. Bialkowski, A. Cavalleri, D. von der Linde, A. Oparin, J. Meyer-ter Vehn, and S. I. Anisimov, Phys. Rev. Lett. 81, 224 (1998).

2 E. Gamaly, Physics Reports 508, 91 (2011).

${ }^{3} \mathrm{P}$. Balling and J. Schou, Reports on Progress in Physics 76, 036502 (2013).

${ }^{4}$ V. Recoules, J. Clérouin, G. Zérah, P. M. Anglade, and S. Mazevet, Phys. Rev. Lett. 96, 055503 (2006).

5 Y. Giret, S. L. Daraszewicz, D. M. Duffy, A. L. Shluger, and K. Tanimura, Phys. Rev. B 90, 094103 (2014).

6 A. Cavalleri, Science 318, 755 (2007).

7 M. E. Povarnitsyn, K. V. Khishchenko, and P. R. Levashov, Applied Surface Science 255, 5120 (2009).

8 J.-P. Colombier, F. Garrelie, N. Faure, S. Reynaud, M. Bounhalli, E. Audouard, R. Stoian, and F. Pigeon, Journal of Applied Physics 111, 024902 (2012).

9 P. Lorazo, L. J. Lewis, and M. Meunier, Phys. Rev. B 73, 134108 (2006).

10 J.-P. Colombier, P. Combis, E. Audouard, and R. Stoian, New Journal of Physics 14, 013039 (2012).

11 V. V. Zhakhovsky, M. M. Budzevich, N. A. Inogamov, I. I. Oleynik, and C. T. White, Phys. Rev. Lett. 107, 135502 (2011).

12 B. Y. Mueller and B. Rethfeld, Phys. Rev. B 87, 035139 (2013).

13 Applied Surface Science 302, 24 (2014), e-MRS 2013 Symposium V:.

14 A. Giri, J. T. Gaskins, B. M. Foley, R. Cheaito, and P. E. Hopkins, Journal of Applied Physics 117, 044305 (2015).

15 E. Bévillon, J. P. Colombier, V. Recoules, and R. Stoian, Phys. Rev. B 89, 115117 (2014).

16 Z. Lin, L. V. Zhigilei, and V. Celli, Phys. Rev. B 77, 075133 (2008).

17 E. Bévillon, J. P. Colombier, B. Dutta, and R. Stoian, The Journal of Physical Chemistry C. (2015), 10.1021/acs.jpcc.5b02085, doi:10.1021/acs.jpcc.5b02085.

18 S. M. Zharkov, V. S. Zhigalov, and G. I. Frolov, Physics of metals and metallography 81, 328 (1996).

19 Y. Mi, D. Yuan, Y. Liu, J. Zhang, and Y. Xiao, Materials Chemistry and Physics 89, 359 (2005).

20 V. Tzitzios, G. Basina, M. Gjoka, V. Alexandrakis, V. Georgakilas, D. Niarchos, N. Boukos, and D. Petridis, Nanotechnology 17, 3750 (2006).

21 C. Wu, M. S. Christensen, J.-M. Savolainen, P. Balling, and L. V. Zhigilei, Phys. Rev. B 91, 035413 (2015).

22 A. Y. Vorobyev and C. Guo, Journal of Applied Physics 104, 063523 (2008).

${ }^{23}$ F. Garrelie, J.-P. Colombier, F. Pigeon, S. Tonchev, N. Faure, M. Bounhalli, S. Reynaud, and O. Parriaux, Opt. Express 19, 9035 (2011).

24 X. Gonze, B. Amadon, P.-M. Anglade, J.-M. Beuken, F. Bottin, P. Boulanger, F. Bruneval, D. Caliste, R. Caracas, M. Cote, et al., Computer Physics Communications 180, 2582 (2009).

25 P. Hohenberg and W. Kohn, Phys. Rev. 136, B864 (1964).

${ }^{26}$ W. Kohn and L. J. Sham, Phys. Rev. 140, A1133 (1965).

27 N. D. Mermin, Phys. Rev. 137, A1441 (1965).

28 J. P. Perdew, K. Burke, and M. Ernzerhof, Phys. Rev. Lett. 77, 3865 (1996).

29 N. Troullier and J. L. Martins, Phys. Rev. B 43, 1993 (1991).

30 H. J. Monkhorst and J. D. Pack, Phys. Rev. B 13, 5188 (1976).

31 R. J. Birgeneau, J. Cordes, G. Dolling, and A. D. B. Woods, Phys. Rev. 136, A1359 (1964).

32 D. A. Porter, K. E. Easterling, and Y. M. Sherif, Phase Transformations in Metals and Alloys, Third Edition (Revised Reprint) (CRC Press, 2009, 2009).

33 R. M. Wentzcovitch and P. K. Lam, Phys. Rev. B 44, 9155 (1991).

34 E. Kim, M. Nicol, H. Cynn, and C.-S. Yoo, Phys. Rev. Lett. 96, 035504 (2006). 